\title{
Retrorectal endometrioid cyst: a case report
}

\author{
Iraklis E Katsoulis*, Ioannis E Katsoulis
}

\begin{abstract}
Introduction: Developmental cysts are the most common retrorectal cystic lesions in adults, whereas reports of endometrioid cysts in this anatomic location are extremely rare.

Case presentation: A 21-year-old nulliparous Greek woman presented with chronic noncyclic pelvic pain, and a retrorectal cyst was diagnosed. The lesion was resected through a laparotomy and, on histologic examination, was found to be an endometrioid cyst. The treatment was completed with a six-month course of a gonadotropinreleasing hormone analogue. One year after surgery, the woman remained free of symptoms, and pelvic imaging showed no recurrence of the lesion. Reviewing the literature, we found only three previous reports of an endometrioid cyst in this anatomic location.
\end{abstract}

Conclusion: In women of reproductive age, endometriosis must be included in the differential diagnosis of retrorectal cysts.

\section{Introduction}

Endometriosis is the presence of endometriotic tissue in anatomic regions outside the uterus [1]. The mostcommon sites are the ovaries and the fallopian tubes, the uterosacral ligaments, and the lateral pelvic peritoneum. Endometriosis can less commonly be found in laparotomy scars, the vagina, and the rectovaginal septum, and also can involve the wall of the colon and the rectum. This is a report of a rare retrorectal endometrioid cyst that was not contiguous to the rectal wall.

Developmental cysts are the most common retrorectal cystic lesions in adults, whereas reports of endometrioid cysts in this anatomic location are extremely rare [2-4].

\section{Case presentation}

A 21-year-old nulliparous Greek woman complained of chronic noncyclic pelvic pain. Abdominal and vaginal examinations were unremarkable, whereas on rectal examination, a soft extraluminal mass was found posteriorly and left laterally.

The rectal mucosa was normal on rigid rectosigmoidoscopy. A pelvic ultrasound scan revealed a cystic lesion posterior to the middle rectum, and blood tests showed a moderately elevated CA $19-9$ (79IU/ml), whereas all other tumour markers were normal. Computed tomography (CT) of the whole abdomen excluded

\footnotetext{
* Correspondence: hrkats@yahoo.co.uk

White Cross Hospital, 1 Sisini Str, 11528 Athens, Greece
}

other intra-abdominal pathology and provided further information regarding the anatomic relations of the lesion. The cyst lay posterior and left lateral to the middle rectum above the level of the pelvic floor and was contiguous neither to the rectal wall nor to the sacrum (Figure 1). Its maximal diameter was about $7 \mathrm{~cm}$.

After administration of preoperative antibiotic prophylaxis, a laparotomy was undertaken through an infraumbilical midline incision. Moderate bilateral ovarian endometriosis and minor endometriosis of the pelvic peritoneum were found; these were ablated with surgical diathermy. Subsequently, the pelvic peritoneum was opened, and the retrorectal space was carefully dissected to avoid injury of the pelvic nervous plexuses and the hypogastric nerves. The retrorectal cystic lesion was removed intact, and on histologic examination was found to be a suppurated endometrioid cyst.

The patient made an uneventful recovery and was discharged on the third postoperative day. The treatment was completed with a six-month course of a gonadotropin-releasing hormone $(\mathrm{GnRH})$ analogue. One year postoperatively, she remained free of symptoms, and follow-up pelvic imaging showed no recurrence of endometriosis.

\section{Discussion}

Developmental cysts are the most common retrorectal cystic lesions in adults, occurring mostly in middle-aged

\section{()




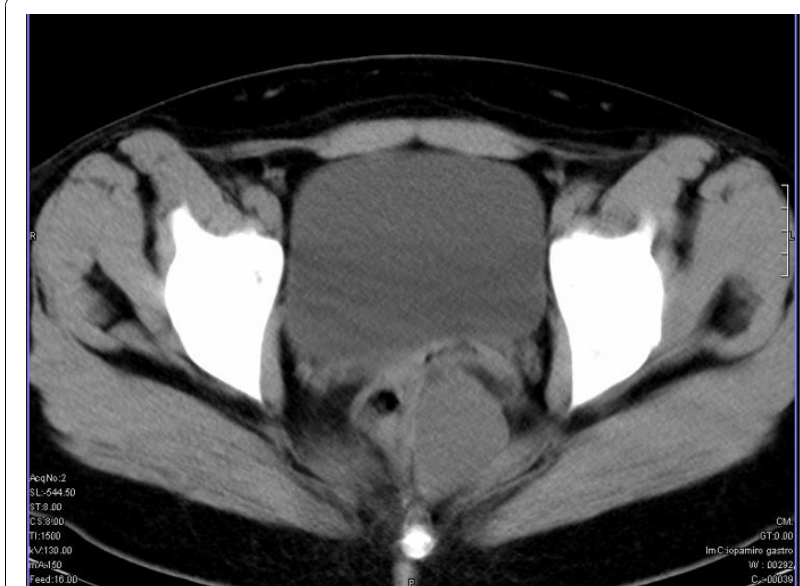

Figure 1 Computed tomography, showing the cystic lesion posterior and left lateral to the middle rectum.

women. They are classified as epidermoid cysts, dermoid cysts, enteric cysts (tailgut cysts or hamartomas and cystic rectal duplication), and neuroenteric cysts, according to their origin and histopathologic features $[5,6]$. The diagnosis of retrorectal cysts can be accomplished with greater than $90 \%$ accuracy with computed tomography (CT) and magnetic resonance imaging (MRI) if the rectum is contrasted $[3,6]$. Such lesions warrant surgical excision to establish the diagnosis and to avoid complications. MRI has been suggested to increase the accuracy of preoperative localization and to enable surgical planning [6]. Transrectal ultrasound, if available, can also be useful in defining the depth of infiltration in cases of rectal involvement [3].

The operative approach can be perineal, abdominal, or combined, depending on the position of the lesion and its anatomic relations with surrounding structures. Retrorectal cysts have been also managed by using a laparoscopic approach [7]. In our patient, the information provided by the CT regarding the size and the anatomic relations of the cyst was considered sufficient, and therefore a pelvic MRI was not performed. We opted to approach the lesion through a laparotomy, aiming to explore her pelvis thoroughly in view of her persistent pelvic pain and elevated CA 19-9 levels.

We found foci of endometriosis on both ovaries and the pelvic peritoneum. A complete resection of the lesion was achieved, and histology made the diagnosis of a suppurated endometrioid cyst. In cases of low perirectal lesions, in which endometriosis is suspected, an alternative strategy can be transperineal excision combined with a laparoscopy for assessment of the intra-abdominal organs. We thought, however, that because the cyst lay posterior and left lateral to the middle rectum, a transperineal approach would neither be sufficient nor warrant the preservation of surrounding structures.

It is not uncommon for endometriosis to involve the rectal wall, requiring an anterior resection of the rectum [8]. Conversely, the presentation of an endometrioid cyst that occupies the retrorectal space, without being contiguous to either the rectal wall or the sacrum, is a rare entity. Reviewing the literature, we found only three previous reports of an endometrioid cyst in this anatomic location [2-4].

\section{Conclusion}

In women of reproductive age, endometriosis must be included in the differential diagnosis of retrorectal cysts.

\section{Consent}

Written informed consent was obtained from the patient for publication of this case report and accompanying images. A copy of the written consent is available for review by the Editor-in-Chief of this journal.

\section{Authors' contributions}

Both authors contributed equally to the writing and read and approved the final manuscript.

\section{Competing interests}

The authors declare that they have no competing interests.

Received: 27 March 2010 Accepted: 30 November 2010 Published: 30 November 2010

\section{References}

1. Farquhar C: Endometriosis. BMJ 2007, 334:249-253.

2. Rieger N, Munday D: Retrorectal endometrial cyst. Arch Gynecol Obstet 2004, 270:67-68.

3. Stroh C, Manger T: Ultrasound diagnosis of rare retrorectal tumours. Zentralbl Chir 2003, 128:1075-1079.

4. Singer MA, Cintron JR, Martz JE, Schoetz DJ, Abcarian H: Retrorectal cyst: a rare tumor frequently misdiagnosed. J Am Coll Surg 2003, 196:880-886.

5. Dahan H, Arrivé L, Wendum D, le Pointe HD, Tubiana JM: Retrorectal developmental cysts in adults: clinical and radiologic-histopathologic review: differential diagnosis, and treatment. Radiographics 2001, 21:575-584

6. Woodfield JC, Chalmers AG, Phillips N, Sagar PM: Algorithms for the surgical management of retrorectal tumours. Br J Surg 2008, 95:214-221.

7. Gunkova P, Martinek L, Dostalik J, Gunka I, Vavra P, Mazur M": Laparoscopic approach to retrorectal cyst. World J Gastroenterol 2008, 14:6581-6583.

8. Brouwer R, Woods RJ: Rectal endometriosis: results of radical excision and review of published work. A N Z J Surg 2007, 77:562-571.

\section{doi:10.1186/1752-1947-4-389}

Cite this article as: Katsoulis and Katsoulis: Retrorectal endometrioid cyst: a case report. Journal of Medical Case Reports 2010 4:389. 